Research Paper

\title{
Effect of YAP Inhibition on Human Leukemia HL-60 Cells
}

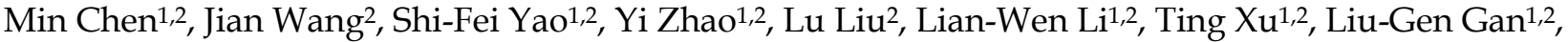 \\ Chun-Lan Xiao ${ }^{1,2}$, Zhi-Ling Shan ${ }^{2}$, Liang Zhong ${ }^{2 \bowtie}$, Bei-Zhong Liu ${ }^{1,2 \bowtie}$ \\ 1. Central Laboratory of Yong-chuan Hospital, Chongqing Medical University, Chongqing, 402160, China \\ 2. Key Laboratory of Laboratory Medical Diagnostics, Ministry of Education, Department of Laboratory Medicine, Chongqing Medical University, \\ Chongqing, 400016, China
}

$\triangle$ Corresponding authors: Liang Zhong, Key Laboratory of Laboratory Medical Diagnostics, Ministry of Education, Department of Laboratory Medicine, Chongqing Medical University, 1\# Yixueyuan Road, Chongqing 400016, China. Tel: +86 13637931208; E-mail: cnmed1@hotmail.com; or Bei-Zhong Liu, Department of Laboratory Medicine, Chongqing Medical University, 1\#, Yixueyuan Road, Chongqing, 400016, China. Tel: +86 18716474304, Fax: +86 023-68485006; E-mail: liubeizhong@cqmu.edu.cn

(c) Ivyspring International Publisher. This is an open access article distributed under the terms of the Creative Commons Attribution (CC BY-NC) license (https://creativecommons.org/licenses/by-nc/4.0/). See http://ivyspring.com/terms for full terms and conditions.

Received: 2017.03.07; Accepted: 2017.05.17; Published: 2017.07.20

\begin{abstract}
Background: Yes-associated protein (YAP), the nuclear effector of the Hippo pathway, is a candidate oncoprotein and participates in the progression of various malignancies. However, few reports have examined the effect of YAP inhibition in human leukemia HL-60 cells.

Methods: We examined the effects of YAP knockdown or inhibition using short hairpin RNA (shRNA) or verteporfin (VP), respectively. Western blot assays were used to determine the expression levels of YAP, Survivin, cyclinDI, PARP, Bcl-2, and Bax. Cell proliferation was assessed using the cell counting kit (CCK-8) assay. Cell cycle progression and apoptosis were evaluated by flow cytometry, and apoptotic cell morphology was observed by Hoechst 33342 staining.

Results: Knockdown or inhibition of YAP led to cell cycle arrest at the G0/G1 phase and increased apoptosis, inhibited cell proliferation, increased levels of Bax and cleaved PARP, and decreased levels of PARP, Bcl-2, Survivin, and cyclinDI. Moreover, Hoechst 33342 staining revealed increased cell nuclear fragmentation.

Conclusion: Collectively, these results show that inhibition of YAP inhibits proliferation and induces apoptosis in HL-60 cells. Therefore, a novel treatment regime involving genetic or pharmacological inhibition of YAP could be established for acute promyelocytic leukemia.
\end{abstract}

Key words: Yes-associated protein, human leukemia HL-60 cells, shRNA, verteporfin, proliferation, apoptosis

\section{Introduction}

The first attempt to standardize the classification of acute myeloid leukemia was undertaken by the French, American, British group, which used morphological analyses and cytochemistry to characterize AML into six subtypes (M1 to M6) [1]. Acute promyelocytic leukemia (APL) is a sub-type of AML (M3). APL is characterized by the $t(15 ; 17)$ translocation, which fuses the promyelocytic leukemia (PML) gene to the retinoic acid receptor a (RARa) gene, and leads to the production of the PML/RARa fusion protein [2]. The HL-60 cell line is one of APL representative cell lines [3]. Patients with
APL can develop serious blood clotting or bleeding problems and children with APL have a high morbidity rate, looking for new treatment targets important $[4,5]$. Clinically, there are two therapeutic agents used for the treatment of APL, all-trans retinoic acid (ATRA) and arsenic trioxide (ATO), which induce differentiation of and promote apoptosis in APL cells, respectively [6, 7]. The use of these drugs has greatly improved the prognosis for patients with $\mathrm{APL}$, and the complete remission rate is now over $90 \%$. However, treatment with ATRA and ATO is not suitable for $10-30 \%$ of patient with APL [8]. 
Therefore, it is crucial to explore new treatment strategies for APL.

YAP is an essential component of the Hippo pathway, which plays important roles in controlling organ size, regulating gene expression in response to changes in differentiation, and in the self-renewal of stem cells [9-11]. YAP, the mammalian ortholog of Drosophila Yorkie, is the downstream effector of the Hippo pathway [12]. Recently, several studies have documented the oncogenic effects of YAP [13-18]. Additionally, YAP amplification and overexpression have been observed in various human cancers, including pancreatic cancer, renal cell carcinoma, breast cancer, cholangiocarcinoma, and medulloblastoma [14, 19-22]. Moreover, YAP expression is significantly higher in patients with leukemia, including chronic lymphoblastic leukemia, and chronic myeloid leukemia than in healthy donors $[15,23]$. Furthermore, it has been suggested that YAP may be a target for regenerative medicine and cancer treatment [24]. Therefore, we wished to examine the role of YAP in the pathogenesis of APL.

In this study, we found that knockdown of YAP inhibited proliferation and induced apoptosis in HL-60 cells. Importantly, we also found that VP-mediated YAP inhibition significantly increased apoptosis and slowed the rate of cell proliferation in HL-60 cells. Taken together, these results suggest that YAP is a novel potential therapeutic target for APL.

\section{Materials and methods}

\section{Cell line and culture}

HL-60 cell line was purchased from the Shanghai Institutes for Biological Sciences (Shanghai, China). HL-60 cells were cultured in RPMI-1640 (Gibco) supplemented with $\sim 10 \%$ fetal bovine serum (FBS; Gibco, Grand Island, NY, USA), $100 \mathrm{U} / \mathrm{mL}$ penicillin and $100 \mu \mathrm{g} / \mathrm{mL}$ streptomycin.

\section{Antibodies}

Following antibodies were used in this study: anti-YAP, anti-PARP, and anti-cyclinD1 (Cell Signaling Technology, USA); anti-Bax, anti-Bcl-2, anti-Survivin (Wanleibio, China); anti- $\beta$-Actin (Zhong shan jin qiao, China).

\section{Transfection}

Lentiviral-mediated short-hairpin RNA (shRNA) was used to this study. The shRNA targeting YAP and the non-targeting shRNA were purchased from Jikai Genechem (Genechem Co.,Ltd. Shanghai, China). shRNA target sequences for YAP: CCGGGCCACC AAGCTAGATAAAGAACTCGAGTTCTTTATCTAG CTTGGTGGCTTTTTG. shRNA non-targeting sequences for negative Control (NC): TTCTCCGAACGTG
TCACGT. HL-60 cells in the logarithmic growth phase $\left(1 \times 10^{5} /\right.$ well $)$ were seeded in a 24 -well plate. These cells were transfected with the GFP-expressing lentiviral vector NC and shRNA-YAP and $1 \mu \mathrm{g} / \mathrm{mL}$ polybrene (Genepharma) was added. After culture for $24 \mathrm{~h}$, the medium was refreshed. Fluorescence was detected following $72 \mathrm{~h}$ of incubation using the fluorescence microscope. The lentiviral YAP-shRNA and lentiviral vector NC-shRNA transfected HL-60 cells were screened with puromycin (Sigma-Aldrich, St. Louis, MO, USA) and successful transfectants were used for subsequent experiments. A fluorescence microscope $(\times 20)$ was used to observe the expression of GFP. There were two groups in this experiment: HL-60/shRNA-NC group and Hl-60/YAP-shRNA group.

\section{Inhibitor of YAP}

VP acts as a YAP inhibitor by blocking the association between TEAD and YAP [25]. VP purchased from Selleck (Selleckchem, Shanghai, China). VP was dissolved in dimethyl sulfoxide (DMSO). HL-60 cells were seed in different concentration VP for $24 \mathrm{~h}$, and the DMSO treatment is control group.

\section{Cell viability assay}

The Cell Counting Kit-8 (CCK-8) assay (7Sea Biotech, Shanghai, China) was used to test cell viability. Cells in each group $\left(1 \times 10^{4} /\right.$ well $)$ were seeded in 96-well plates and incubated. In brief, $10 \mu \mathrm{L}$ of CCK-8 (7Sea Cell Counting Kit; Sevenseas Futai Biotechnology Co., Ltd., Shanghai, China) was added to each well followed by incubation for $2 \mathrm{~h}$ at $37^{\circ} \mathrm{C}$. The cell viability was assessed by detection of absorbance at $450 \mathrm{~nm}$ using a spectrophotometer. The experiment was repeated at least three times.

\section{RNA isolation and RT-PCR}

Total RNA was extracted from cells in each group using Trizol reagent, as per manufacturer's instructions (Invitrogen, Carlsbad, California). The first-strand cDNA was synthesized from $1 \mu \mathrm{g}$ of total RNA using a Prime Script Kit (TAKARA, Dalian, China). YAP gene expression was tested by reverse transcriptase polymerase chain reaction (RT-PCR) with cDNA Synthesis Kit (TAKARA, Dalian, China). $\beta$-Actin was used as an endogenous control. All samples were run in duplicate for each experiment. Do $1 \%$ agarose gel lelctrophoresis as soon as we acquire the PCR product. And $5 \mu \mathrm{L}$ PCR product was used in each lane. Gene expression analysis was performed with the Quantity One Software (BIO-RAD, USA). The PCR conditions were: pre-denaturation at $95{ }^{\circ} \mathrm{C}$ for $5 \mathrm{~min}, 35$ cycles of denaturation at $95^{\circ} \mathrm{C}$ for $30 \mathrm{~s}$, annealing at $64^{\circ} \mathrm{C}$ for 30 
$\mathrm{s}$, and extension at $72{ }^{\circ} \mathrm{C}$ for $100 \mathrm{~s}$, and a final extension at $72{ }^{\circ} \mathrm{C}$ for $5 \mathrm{~min}$. The amplification of $\beta$-Actin gene was the as for YAP. The mRNA expression levels of the target gene were normalized to those of $\beta$-Actin. The specific primers for YAP were 5'- TGAACAAACGTCCAGCAAGATAC-3' (forward) and 5'- CAGCCCCCAAAATGAACAGTAG-3' (reverse). Those for $\beta$-Actin were 5'-CACCACACCTTCT ACAATGAGC-3' (forward) and 5'-GTGATCTCCTT CTGCATCCTGT-3' (reverse).

\section{Western blot analysis}

Protein concentration was determined with BCA method. A total of $50 \mu \mathrm{g}$ of protein was added in $10 \%$ sodium dodecyl sulfate-polyacrylamide gel, and then transferred to polyvinylidene difluoride membrane. The membrane was blocked with 5\% non-fat milk for $2 \mathrm{~h}$, then incubated with specific antibodies (monoclonal) antibody overnight at $4{ }^{\circ} \mathrm{C}$, followed by incubation with HRP-conjugated secondary antibody for $1.5 \mathrm{~h}$ at room temperature. Detection was performed using the enhanced chemiluminescence substrate (ECL) (Millipore, USA). Signals were visualized and analyzed by the Bio-Rad Gel Imaging System on cool image workstation II (Viagene, USA). Each experiment was repeated at least three times.

\section{Hoechst 33342 staining analysis}

Cell apoptosis was analyzed by Hoechst 33342 staining (Apoptosis-Hoechst staining kit; Beyotime Biotechnology, Haimen). Briefly, cells were immersed in $0.5 \mathrm{~mL}$ of methanol for $15 \mathrm{~min}$, followed by rinsing third with PBS. Then cells were stained with $1 \mu \mathrm{g} / \mathrm{mL}$ Hoechst 33342 compounds in a dark chamber at room temperature for $10 \mathrm{~min}$ and again rinsed twice with PBS. Cells were analyzed by fluorescence microscopy. The apoptotic cells are seen as pyknotic and have fragmented nuclei emitting intense fluorescence $(\times 20)$. The experiment was repeated at least three times.

\section{Flow cytometric assay}

Cells were washed using PBS. And the cell pellets were resuspended and stained with annexin V-FITC and propidium iodide (PI) (Sigma-Aldrich). The rate of cell apoptosis was analyzed using a FACsorter (BD Biosciences, San Jose, CA, USA) after incubation for $15 \mathrm{~min}$ at room temperature. For cell cycle detection, cells were fixed with pre-cooled $70 \%$ ethanol overnight at $-20^{\circ} \mathrm{C}$. After centrifugation, the cells were resuspended with RNase solution in a $37^{\circ} \mathrm{C}$ water bath for $30 \mathrm{~min}$. Then propidium iodide staining solution was added and incubated for $30 \mathrm{~min}$ in the dark at room temperature. The cell cycle distribution was determined using a FACsorter. And for the transfection efficiency also was tested by flow cytometric. Each experiment was repeated at least three times.

\section{Statistical analysis}

Values are expressed as the mean \pm standard deviation. Statistical analysis was performed using SPSS 17.0 software (SPSS, Inc., Chicago, IL, USA). An independent samples t-test was employed for comparing the means between two groups. $\mathrm{P}<0.05$ was considered to indicate a statistically significant difference. Each experiment was repeated at least three times.

\section{Results}

\section{Lentivirus-mediated YAP knockdown in human leukemia HL-60 cells}

The YAP-shRNA was introduced to HL-60 cells to silence YAP expression. Cells containing YAP-shRNA were identified by GFP fluorescence and accounted for approximately $\sim 80 \%$ of the cell population (Figure 1A-B). RT-PCR assay showed that mRNA level of YAP significantly lowered in the sh-YAP (YAP-knockdown HL-60 cells) group compared with NC (negative control HL-60 cells) group (Figure 1C). Meanwhile, western blot analysis also showed that YAP protein expression was knocked down in these cells $(P<0.05)$ (Figure 1D-E).

\section{The effect of YAP knockdown on proliferation in human leukemia HL-60 cells}

We used the CCK-8 assay to assess the effect of YAP on HL-60 cell proliferation. Our results show that, compared with that observed in NC group cells, the proliferation of HL-60/sh-YAP cells was significantly inhibited in a time-dependent manner ( $P$ $<0.05$ ) (Figure 2A). Additionally, the expression level of Survivin was significantly lower in YAP-knockdown HL-60 cells than in control cells ( $\mathrm{P}<$ 0.05 ) (Figure 2B-C). Further, we tested the cycle distribution by FCM. Knockdown of YAP in HL-60 cells dramatically increased the percentage of cells in the G0/G1 phase from $36.45 \%$ to $53.24 \%$ (Figure 2D). Next, we detected the cycle-related protein, cyclinD1, by western blot. Knockdown of YAP decreased the level of cyclinD1 protein in HL-60 cells (Figure 2E-F). These data provide strong evidence that YAP knockdown inhibits HL-60 cell proliferation by causing G0/G1 phase cell-cycle arrest.

\section{Promotion of apoptosis by YAP knockdown in HL-60 cells}

Our FCM results show that YAP knockdown promotes apoptosis in HL-60 cells (Figure 3A). Furthermore, morphological changes characteristic of apoptosis were observed in YAP knockdown HL-60 cells (Figure 3B). Western blot analysis showed that 
the expression levels of PARP and Bcl-2 proteins, associated with apoptosis, were decreased, while cleaved PARP and Bax were increased in YAP silenced HL-60 cells (Figure 3C-G). These results indicated that YAP knockdown triggers apoptosis in HL-60 cells via regulating the expression of apoptosis-related proteins.

\section{The VP YAP inhibitor suppresses YAP expression in HL-60 cells}

RT-PCR and Western blot assay showed that mRNA and protein expression of YAP were significantly lowered in VP-mediated YAP inhibition group when compared with DMSO treatment group cells $(\mathrm{P}<0.05)$ (Figure 4).

\section{VP inhibits proliferation of HL-60 cells}

Using the CCK-8 assay to assess cell proliferation activity, we observed that treatment with different VP concentrations $(0-20 \mu \mathrm{M})$ for $24 \mathrm{~h}$ resulted in a dose-dependent reduction in cell viability (Figure 5A). Based on these observations, $10 \mu \mathrm{M}$ VP was chosen for further analyses of YAP inhibition. In addition to inhibiting YAP expression, VP also inhibited the protein expression of Survivin (Figure 5B-C). These data suggested that VP inhibits proliferation in HL-60 cells.

A

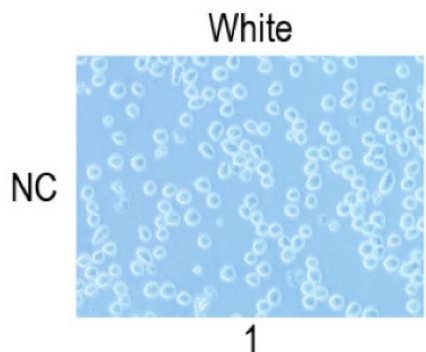

1

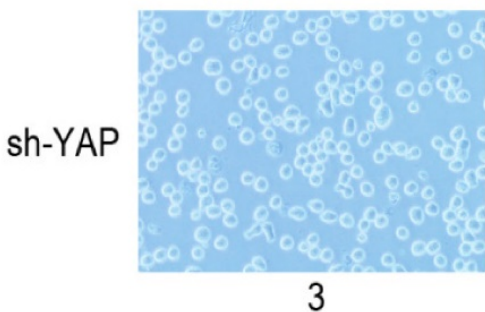

B

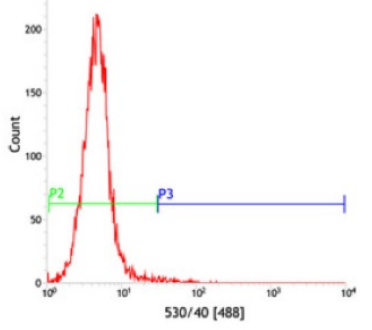

untreated cells

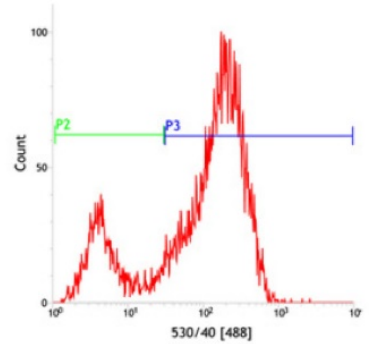

NC
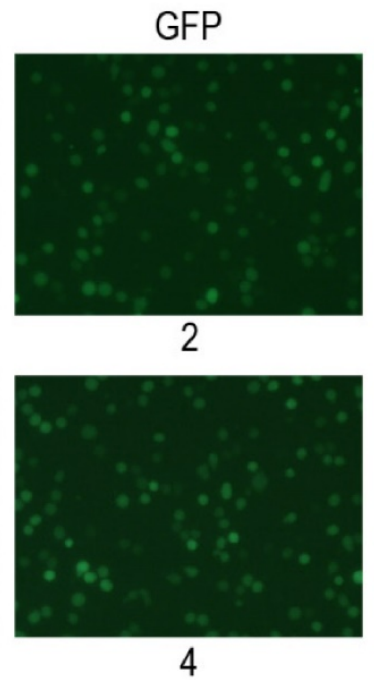

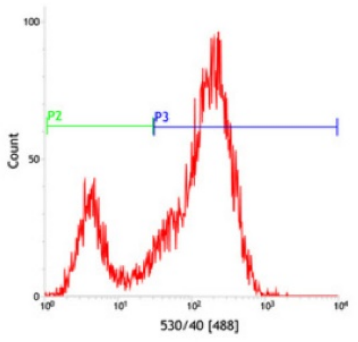

sh-YAP

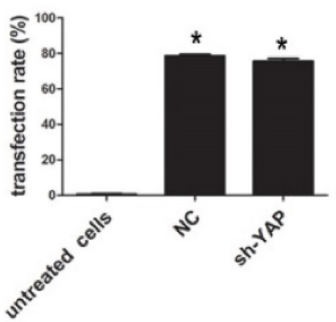

E

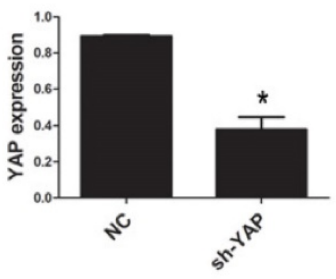

Figure 1. Knock down YAP of HL-60 cell. (A) 1 and 3, light microscopy; 2 and 4, fluorescent microscopy. 1 and 2 images of HL-60 cells were transfected with negative control lentivirus; 3 and 4 images of HL-60 cells were transfected with YAP-shRNA $(\times 20)$. (B) The transfection efficiency was tested by FCM. (C) The mRNA level of YAP was tested by RT-PCR. (D) The protein expression of YAP was detected by western blot. (E) Quantitative analysis was performed by measuring the relative protein expression level of YAP to $\beta$-Actin. Data are expressed as means $\pm S D$. $* P<0.05$. 
A

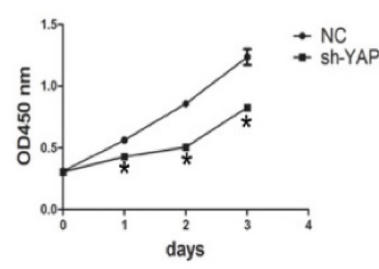

D

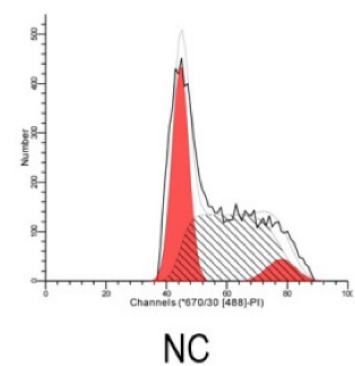

B

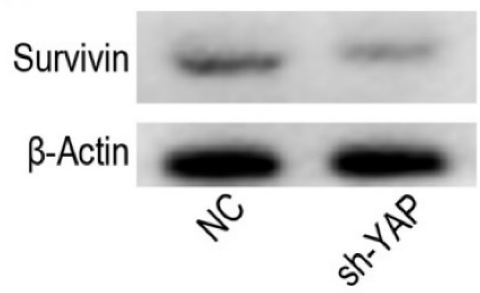

C

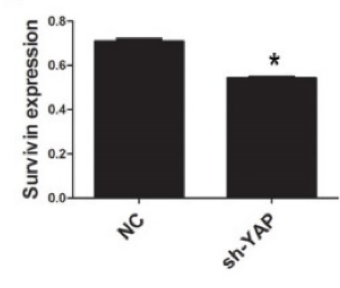

E
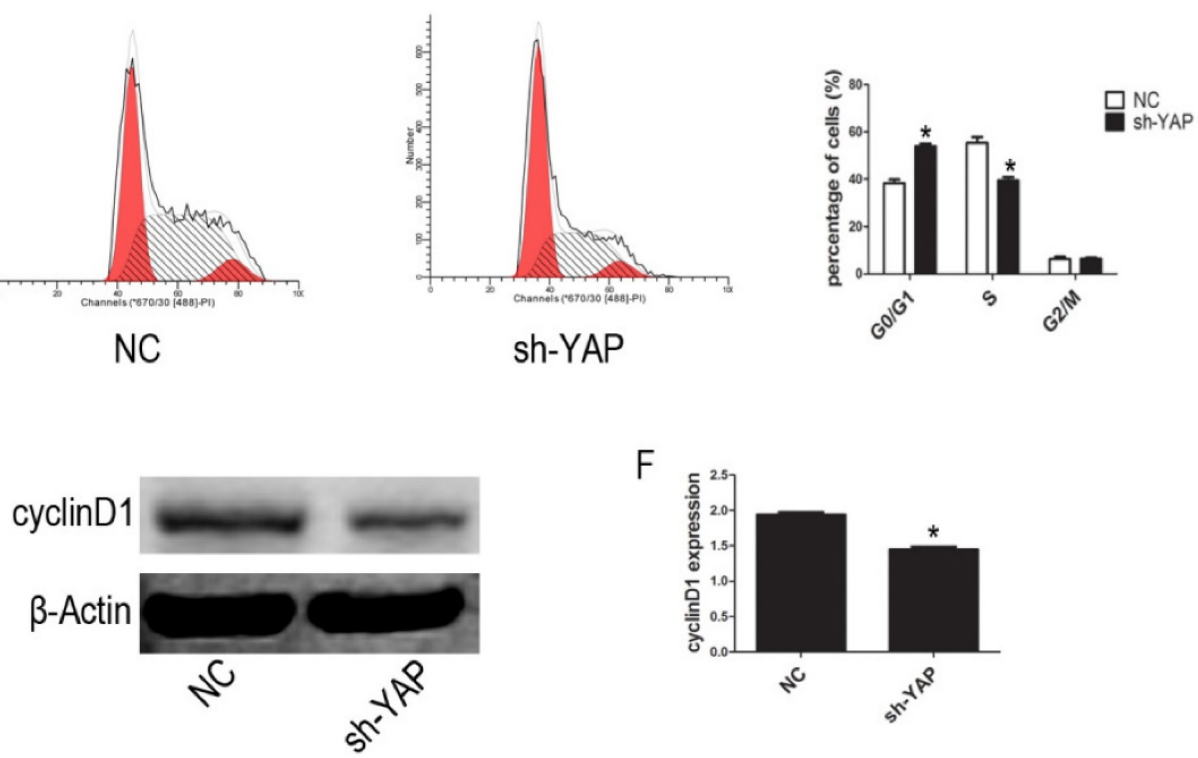

F

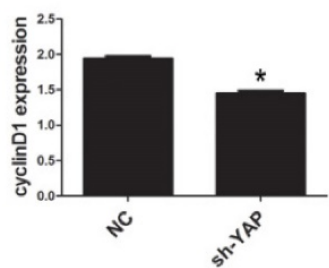

Figure 2. YAP knockdown inhibited proliferation of HL-60 cells. (A) Cells activity was detected by CCK-8 assay. (B) The expression level of Suvivin was tested by western blot. (D) Cell cycle distribution was tested by FCM. (E) The expression level of cyclinDI was determined by western blot analysis. (C and F) Quantitative analysis was performed by measuring the relative protein expression levels of Survivin and cyclinD1 to $\beta$-Actin. Data are expressed as means \pm SD. $* P<0.05$.

\section{VP induced cycle arrest at G0/G1 phase}

To further investigate the VP-induced suppression of cell proliferation, we examined cell cycle distribution by FCM. The percentage of cells arrested the G0/G1 phase of the cell cycle increased from $37.95 \%$ to $52.91 \%$ upon VP treatment (Figure 6A). To reveal the molecular mechanisms involved in YAP inhibition-mediated cell cycle arrest, we analyzed the expression of the cell cycle-related protein, cyclinD1, using western blot assays. Compared with the DMSO treatment group, cell treated with VP exhibited decreased cyclinD1 expression level (Figure 6B-C). These results suggested that VP inhibited cell proliferation by down-regulating cell cycle-related protein expression leading to cell cycle arrest.

\section{VP induced apoptosis in HL-60 cells}

To examine whether VP affects apoptosis in HL-60 cell, we used FCM to quantify apoptotic cells. The FCM results show that the percentage of apoptotic cells increased after VP treatment (Figure 7A). We observed the morphological characteristics of apoptotic cells using Hoechst 33342 staining and observed nuclear fragmentation in the VP treatment group, but not in the DMSO control group (Figure 7B). Additionally, we used western blot analysis to examine the expression levels of apoptosis-related proteins. Compared with the DMSO treatment group, levels of cleaved PARP and Bax increased, and PARP and $\mathrm{Bcl}-2$ decreased in the VP treatment group (Figure 7C-G). These data indicated that VP induces apoptosis in HL-60 cells.

\section{Discussion}

APL is a rare form of cancer, and targeted therapy has successfully eradicated leukemia stem cells in the majority of affected patients. ATRA and ATO lead to complete remission in most patients with APL, but a large proportion of patients eventually experience relapse [2, 26]. Therefore, novel therapeutic targets are necessary to improve the outcomes for patients with APL [27]. YAP functions as an oncoprotein by interacting with TEAD, forming a protein complex critical for the transcription of downstream genes such as c-Myc and Survivin [15, 22]. Recently, porphyrin family members including VP, hematoporphyrin, and protoporphyrin IX have 
been found to abrogate the interaction between YAP and TEAD and found function as YAP inhibitors [25, 28]. However, while MST1/2 and YAP1 gene expression have been analyzed in AML [29], and inhibition of YAP results in a significant anti-leukemia effect in chronic myeloid leukemia [15], the effect of YAP inhibition in APL remains unclear. Here, we demonstrate the effects of YAP knockdown and the inhibition of YAP function by shRNA and VP, respectively, in HL-60 cells. Regrettably, due to a lack of clinical samples and other suitable leukemia cell lines, our analysis was limited to the examination of the effect of YAP inhibition, or knockdown, in human leukemia HL-60 cells. Our study revealed that YAP might be involved in the pathogenesis of APL and could be a potential target for the treatment of APL.
Using a CCK-8 assay, we showed that cell proliferation was significantly inhibited in both YAP knockdown and VP treatment groups, compared with control group. Inhibition of Survivin expression could promote apoptosis in leukemia cells $[27,30]$. Here, we observed that the expression levels of Survivin and cyclinD1decreased in YAP knockdown and VP treatment groups, compared with control groups. Furthermore, the cell cycle in the G0/G1 phase significantly increased in both YAP knockdown and VP treatment groups, compared with control groups. cyclinD1 is a cell cycle-related protein, closely associated with the proliferation of cancer cells, and may promote tumor formation [31]. Therefore, our results suggested that cell proliferation was inhibited in HL-60 cells by inducing cell cycle arrest at the G0/G1 phase.

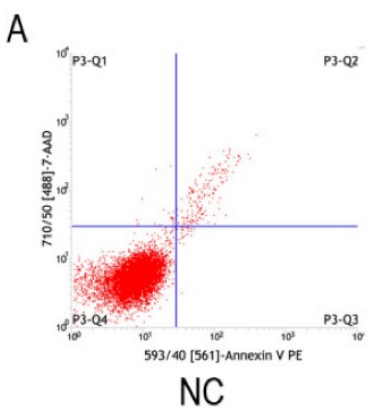

B

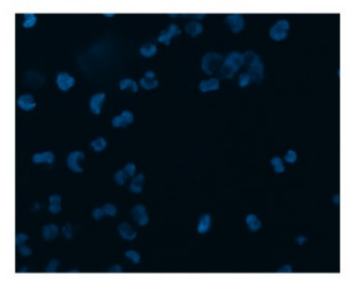

NC
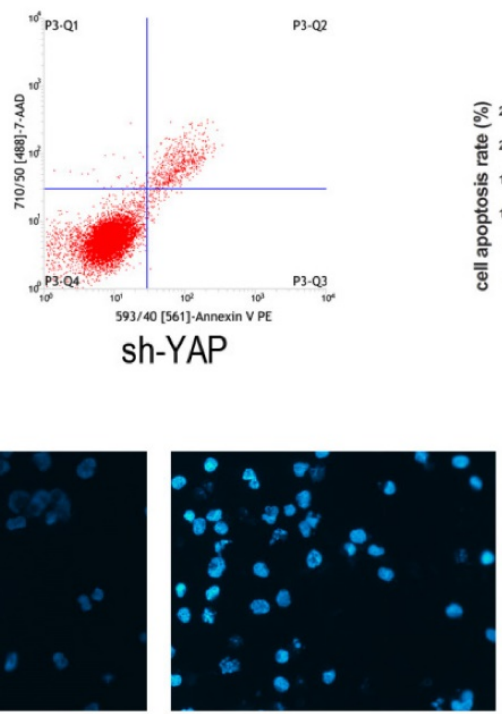

sh-YAP

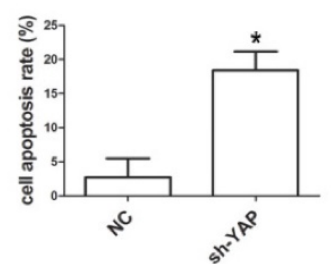

C

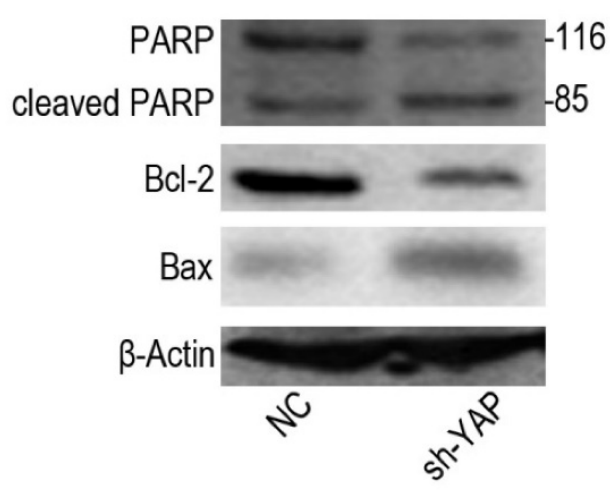

D
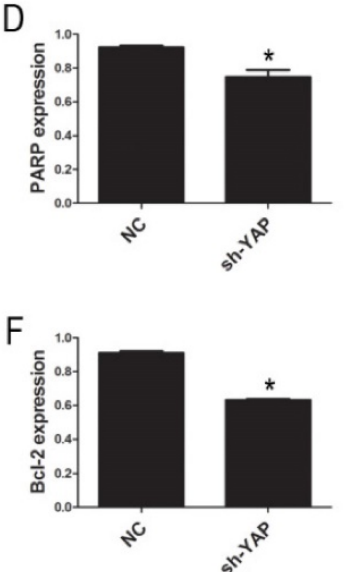

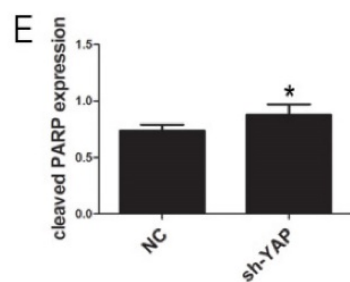

G

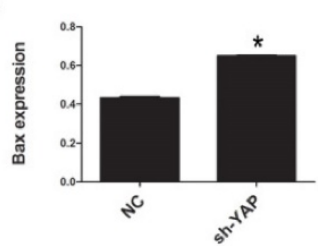

Figure 3. Knockdown YAP induced apoptosis of HL-60 cells. (A) Cells apoptosis was analyzed by FCM using double staining with FITC-labeled annexin-V and propidium iodide. Cells undergoing early apoptosis are Annexin V-FITC+/PI-, whereas cells undergoing late apoptosis are Annexin V-FITC+/PI+. The percentages of late and early apoptotic cells were summed to give the total number of apoptotic cells. (B) Morphological features of the cell apoptosis were observed by Hoechst 33342 staining $(\times 20)$. (C) The expression levels of PARP, cleaved PARP, Bcl-2, and Bax were determined by western blot. (D-G) Quantitative analysis was performed by measuring the relative protein expression levels of PARP, cleaved PARP, Bcl-2, and Bax to $\beta$-Actin. Data are expressed as means \pm SD. $* P<0.05$. 
A

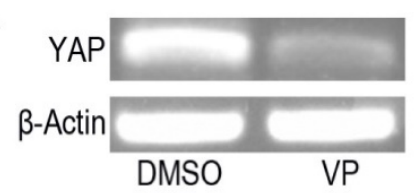

B

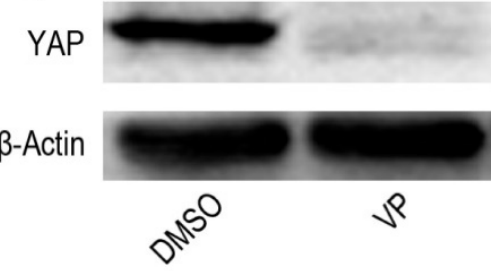

C

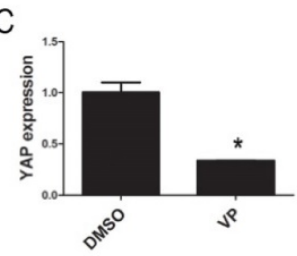

Figure 4. VP inhibits the expression of YAP. (A)The mRNA level of YAP was tested by RT-PCR. (B) The protein expression of YAP was tested by western blot. (C) Quantitative analysis was performed by measuring the relative protein expression level of $Y A P$ to $\beta$-Actin. Data are expressed as means \pm SD. $* P<0.05$.

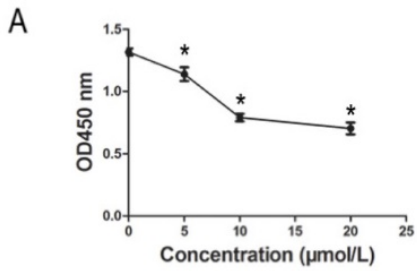

B

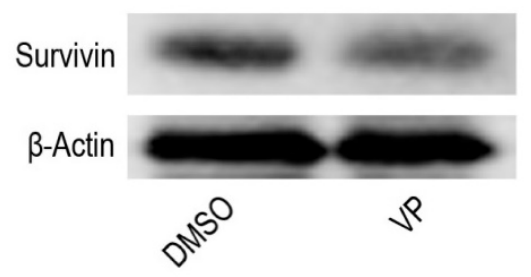

C

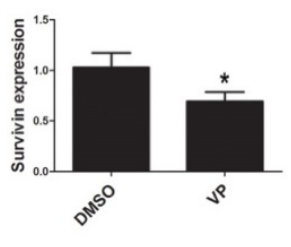

Figure 5. VP inhibited proliferation in HL-60 cells. (A) Cells proliferation was determined by CCK- 8 assay. (B) The expression level of Survivin was tested by western blot. (C) Quantitative analysis was performed by measuring the relative protein expression level of Survivin to $\beta$-Actin. Data are expressed as means \pm SD. $* P<0.05$.

A

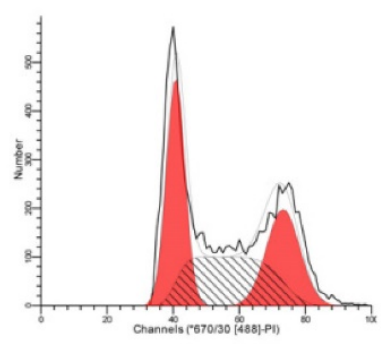

DMSO

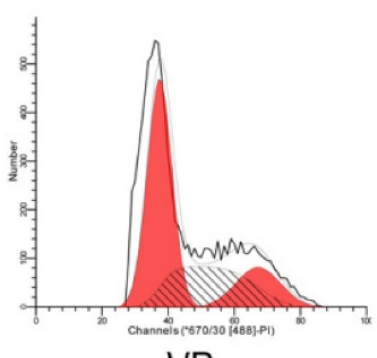

VP

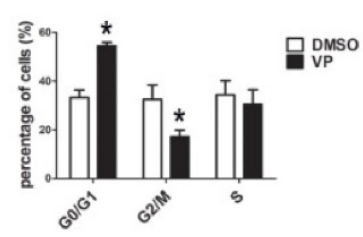

B

cyclinD1

$\beta$-Actin
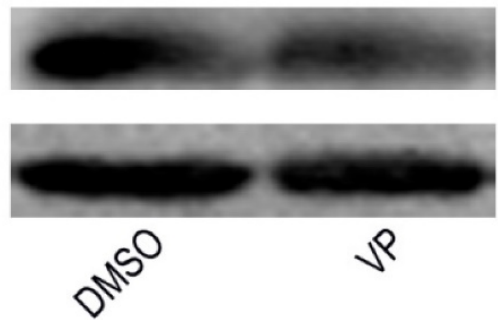

C

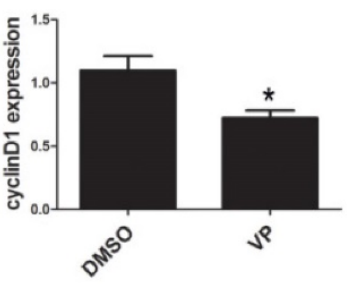

Figure 6. VP affects cell cycle. (A) Cell cycle distribution was tested by FCM. (B) The expression level of cyclinDI was determined by western blot. (C) Quantitative analysis was performed by measuring the relative expression level of cyclinD1 to $\beta$-Actin. Data are expressed as means \pm SD. $* P<0.05$. 
A

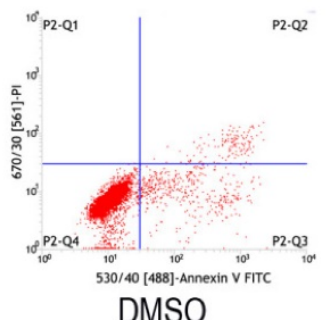

DMSO

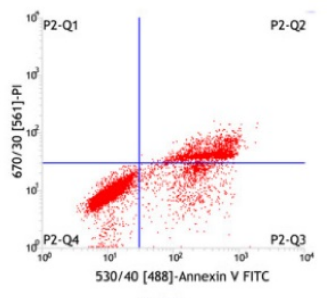

VP

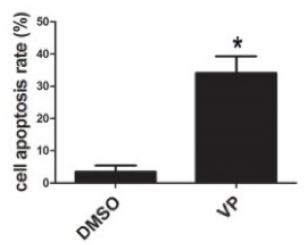

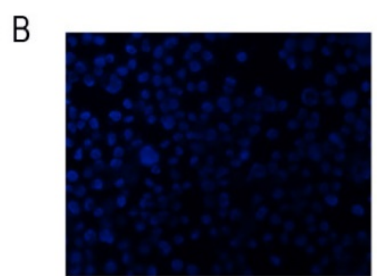

DMSO

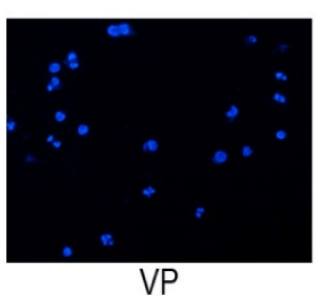

C

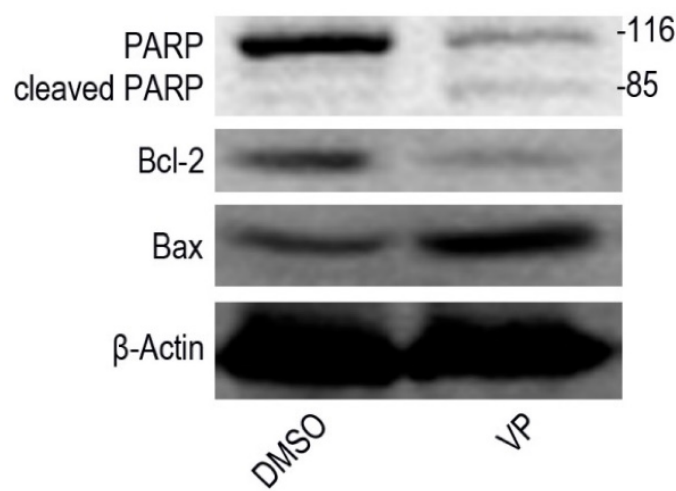

D

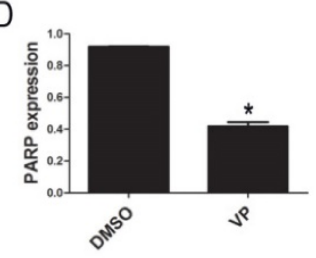

$\mathrm{F}$

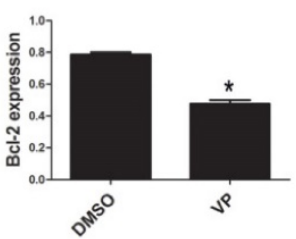

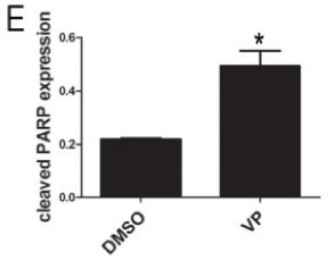

G

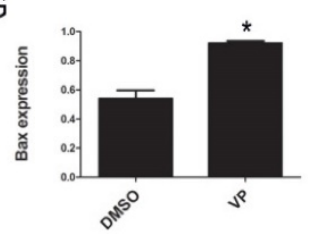

Figure 7. VP induced apoptosis in HL-60 cells. (A) Cells apoptosis was analyzed by FCM. (B) Morphological features of the cell apoptosis were observed by Hoechst 33342 staining $(\times 20)$. (C) The expression levels of PARP, cleaved PARP, Bcl-2, and Bax were determined by western blot. (D-G) Quantitative analysis was performed by measuring the relative protein expression levels of PARP, cleaved PARP, Bcl-2, and Bax to $\beta$-Actin. Data are expressed as means \pm SD. *P $<0.05$.

We used FCM to examine apoptosis and found that YAP knockdown and inhibition significantly increased the percentage of apoptotic HL-60 cells. We observed the morphological characteristics of apoptotic cells using Hoechst 33342 staining and found that nuclear fragmentation, indicative of late stage apoptosis, was easily observed in YAP knockdown or inhibition groups, but not in NC or DMSO treated groups. Additionally, we observed significantly increased levels of cleaved PARP and Bax, and decreased levels of Bcl-2 and PARP following knockdown of YAP by shRNA, or inhibition of YAP function using VP. These results suggest that knockdown of YAP, by shRNA or VP-mediated inhibition of YAP function, induces apoptosis through regulating the expression levels of apoptosis-related proteins.

In conclusion, knockdown of YAP by shRNA or inhibition of the function of YAP using VP, impedes cell proliferation and induces apoptosis in HL-60 cells. Therefore, YAP might be a potential new target for the treatment of APL.

\section{Abbreviations}

AML: acute myeloid leukemia; APL: acute promyelocytic leukemia; ATO: arsenic trioxide; ATRA: all-trans retinoic acid; CCK-8: cell counting kit; DMSO: dimethyl sulfoxide; FCM: flow cytometry; PML: promyelocytic leukemia; RARa: retinoic acid receptor a; shRNA: short hairpin RNA; VP: verteporfin; YAP: Yes-associated protein; RT-PCR: reverse transcriptase polymerase chain reaction.

\section{Acknowledgement}

Our study was supported by the National Natural Science Foundation of China (No. 81171658) and the Natural Science Foundation Project of CQ CSTC (grant No. 2011BA5037). 


\section{Competing Interests}

The authors have declared that no competing interests exist.

\section{References}

1. Thuler LC, Pombo-de-Oliveira MS. Acute promyelocytic leukaemia is highly frequent among acute myeloid leukaemias in Brazil: a hospital-based cancer registry study from 2001 to 2012. Annals of hematology. 2017; 96: 355-62.

2. Varghese L, Janckila A, Yam LT. Acute promyelocytic leukemia. New methods in diagnosis and treatment. The Journal of the Kentucky Medical Association. 1999; 97: 61-5.

3. Miyoshi Y, Nakamura H, Tagami T, Sasaki S, Nakao K. 3,5,3'-Triiodothyronine stimulates retinoic acid-induced differentiation in HL-60 cells. Molecular and cellular endocrinology. 1994; 103: 119-23.

4. Farah RA, Horkos JG, Bustros YD, Farhat HZ, Abla O. A Multicenter Experience from Lebanon in Childhood and Adolescent Acute Myeloid Leukemia: High rate of Early Death in Childhood Acute Promyelocytic Leukemia. Mediterranean journal of hematology and infectious diseases. 2015; 7: e2015012.

5. Takahashi H, Watanabe T, Kinoshita A, Yuza Y, Moritake H, Terui K, et al. High event-free survival rate with minimum-dose-anthracycline treatment in childhood acute promyelocytic leukaemia: a nationwide prospective study by the Japanese Paediatric Leukaemia/Lymphoma Study Group. British journal of haematology. 2016; 174: 437-43.

6. Reynolds CP, Lemons RS. Retinoid therapy of childhood cancer. Hematology/oncology clinics of North America. 2001; 15: 867-910.

7. Slack JL, Waxman S, Tricot G, Tallman MS, Bloomfield CD. Advances in the management of acute promyelocytic leukemia and other hematologic malignancies with arsenic trioxide. The oncologist. 2002; 7 Suppl 1: 1-13.

8. Vassilakopoulos TP, Asimakopoulos JV, Plata E, Kelepesis G, Petevi K, Koutsi $\mathrm{C}$, et al. Recurrent acute myopericarditis without effusion during ATRA induction and ATO salvage of APL: a variant form of the differentiation syndrome? Leukemia \& lymphoma. 2016: 1-4.

9. Chung H, Lee BK, Uprety N, Shen W, Lee J, Kim J. Yap1 is dispensable for self-renewal but required for proper differentiation of mouse embryonic stem (ES) cells. EMBO reports. 2016; 17: 519-29.

10. Hsiao C, Lampe M, Nillasithanukroh S, Han W, Lian X, Palecek SP. Human pluripotent stem cell culture density modulates YAP signaling. Development (Cambridge, England). 2016; 11: 662-75.

11. Huang Z, Hu J, Pan J, Wang Y, Hu G, Zhou J, et al. YAP stabilizes SMAD1 and promotes BMP2-induced neocortical astrocytic differentiation. Development. 2016; 143: 2398-409.

12. Huang J, Wu S, Barrera J, Matthews K, Pan D. The Hippo signaling pathway coordinately regulates cell proliferation and apoptosis by inactivating Yorkie, the Drosophila Homolog of YAP. Cell. 2005; 122: 421-34.

13. Heidary Arash E, Attisano L. A role for Hipk in the Hippo pathway. Science signaling. 2013; 6: pe18.

14. Fernandez LA, Squatrito M, Northcott P, Awan A, Holland EC, Taylor MD, et al. Oncogenic YAP promotes radioresistance and genomic instability in medulloblastoma through IGF2-mediated Akt activation. Oncogene. 2012; 31: 1923-37.

15. Li H, Huang $Z$, Gao M, Huang $N$, Luo Z, Shen $H$, et al. Inhibition of $Y A P$ suppresses CML cell proliferation and enhances efficacy of imatinib in vitro and in vivo. Journal of experimental \& clinical cancer research : CR. 2016; 35: 134.

16. Bae JS, Kim SM, Lee H. The Hippo signaling pathway provides novel anti-cancer drug targets. Oncotarget. 2016.

17. Liao T, Wen D, Ma B, Hu JQ, Qu N, Shi RL, et al. Yes-associated protein 1 promotes papillary thyroid cancer cell proliferation by activating the ERK/MAPK signaling pathway. Oncotarget. 2016.

18. Murakami S, Shahbazian D, Surana R, Zhang W, Chen H, Graham GT, et al. Yes-associated protein mediates immune reprogramming in pancreatic ductal adenocarcinoma. Oncogene. 2017; 36: 1232-44.

19. Cao JJ, Zhao XM, Wang DL, Chen KH, Sheng $X, \mathrm{Li} W B$, et al. YAP is overexpressed in clear cell renal cell carcinoma and its knockdown reduces cell proliferation and induces cell cycle arrest and apoptosis. Oncology reports. 2014; 32: 1594-600

20. Li X, Liu Y, Zhang C, Niu Q, Wang H, Che C, et al. Stiehopus japonieus acidic mucopolysaccharide inhibits the proliferation of pancreatic cancer SW1990 cells through Hippo-YAP pathway. Oncotarget. 2017.

21. Kim HM, Jung WH, Koo JS. Expression of Yes-associated protein (YAP) in metastatic breast cancer. International journal of clinical and experimental pathology. 2015; 8: 11248-57.

22. Pei $\mathrm{T}$, Li $\mathrm{Y}$, Wang J, Wang $\mathrm{H}$, Liang $\mathrm{Y}$, Shi $\mathrm{H}$, et al. $\mathrm{YAP}$ is a critical oncogene in human cholangiocarcinoma. Oncotarget. 2015; 6: 17206-20.

23. Yu Z, Yi S, Zhang Y, Li Z, Qiu L. [Expression of LATS mRNA in mantle cell lymphoma and its clinical significance]. Zhonghua yi xue za zhi. 2015; 95: $3285-8$.

24. Santucci M, Vignudelli T, Ferrari S, Mor M, Scalvini L, Bolognesi ML, et al. The Hippo Pathway and YAP/TAZ-TEAD Protein-Protein Interaction as Targets for Regenerative Medicine and Cancer Treatment. Journal of medicinal chemistry. 2015; 58: 4857-73.

25. Liu-Chittenden Y, Huang B, Shim JS, Chen Q, Lee SJ, Anders RA, et al. Genetic and pharmacological disruption of the TEAD-YAP complex suppresses the oncogenic activity of YAP. Genes Dev. 2012; 26: 1300-5.

26. Lou Y, Ma Y, Sun J, Ye X, Pan H, Wang Y, et al. Evaluating frequency of PML-RARA mutations and conferring resistance to arsenic trioxide-based therapy in relapsed acute promyelocytic leukemia patients. Annals of hematology. 2015; 94: 1829-37.

27. Smith AM, Little EB, Zivanovic A, Hong P, Liu AK, Burow R, et al. Targeting survivin with YM155 (Sepantronium Bromide): a novel therapeutic strategy for paediatric acute myeloid leukaemia. Leukemia research. 2015; 39: 435-44.

28. Gibault F, Corvaisier M, Bailly F, Huet G, Melnyk P, Cotelle P. Non-Photoinduced Biological Properties of Verteporfin. Current medicinal chemistry. 2016; 23: 1171-84.

29. Safari S, Movafagh A, Zare-Adollahi D, Ghadiani M, Riazi-Isfahani S, Safavi-Naini N, et al. MST1/2 and YAP1 gene expression in acute myeloid leukemia. Leukemia \& lymphoma. 2014; 55: 2189-91.

30. Sasaki R, Ito S, Asahi M, Ishida Y. YM155 suppresses cell proliferation and induces cell death in human adult T-cell leukemia/lymphoma cells. Leukemia research. 2015; 39: 1473-9.

31. Nuzzo AM, Giuffrida D, Masturzo B, Mele P, Piccoli E, Eva C, et al. Altered expression of G1/S phase cell cycle regulators in placental mesenchymal stromal cells derived from preeclamptic pregnancies with fetal-placental compromise. Cell cycle (Georgetown, Tex). 2017; 16: 200-12. 